\title{
RECUPERAÇÃO DA TOPOGRAFIA DE OVOS POR MEIO DA TÉCNICA DE MOIRÉ E CALIBRAÇÃO INDEPENDENTE
}

\author{
GLEICE C. DE A. E SILVA ${ }^{1}$, TADAYUKI YANAGI JÚNIOR ${ }^{2}$, EBERSON SILVA $^{3}$, \\ ROBERTO A. BRAGA JÚNIOR ${ }^{4}$, ALESSANDRO T. CAMPOS ${ }^{5}$
}

\begin{abstract}
RESUMO: A recuperação da topografia de ovos por meio de análise de imagens digitais permite, de forma não destrutiva, a estimativa das massas da gema, da clara, da casca e totais, que são produtos amplamente utilizados na esfera industrial. O presente trabalho buscou desenvolver uma metodologia para a recuperação da forma tridimensional de ovos por meio de análise de imagens, em especial usando a técnica de moiré. Foram utilizados ovos de diferentes tamanhos, classificados em: XL (mais de $73 \mathrm{~g}$ ), L (63 a $73 \mathrm{~g}$ ), M (53 a $63 \mathrm{~g}$ ) e $\mathrm{S}$ (até $53 \mathrm{~g}$ ). Em cada teste realizado em laboratório, um ovo, aleatoriamente selecionado, foi iluminado com a projeção de grades, e as imagens foram capturadas e processadas para a obtenção da forma tridimensional do objeto pela técnica de moiré (TM). As dimensões dos seus eixos principais foram medidas por meio de um paquímetro, sendo usadas como referência para calibração da TM. Equações empíricas foram ajustadas para a correção das dimensões, apresentando coeficiente de determinação de 0,$961 ; 0,955$ e 0,988. A TM mostrou-se adequada para a recuperação da topografia de ovos, proporcionando erros menores ou iguais a 5,47\%, com a clara necessidade de uma calibração independente, nas três dimensões, e sem a necessidade de usar um objeto calibrador.
\end{abstract}

PALAVRAS-CHAVE: análise de imagens, ovo, forma tridimensional.

\section{EGGS' TOPOGRAPHY RECOVERY BY MEANS OF THE MOIRÉ TECHNIQUE AND BY AN INDEPENDENT CALIBRATION}

\begin{abstract}
The recovery of the topography of eggs using digital images analysis allows the prediction with a non-destructive and with a non-contact way of the egg mass, yolk, egg white, and eggshell, which are products widely used in the industrial sphere. The present research seeks to develop a methodology to recovery the tridimensional shape of eggs through image analysis, in particular by the moiré technique with the proposition of an independent calibration. For the accomplishment of this research, eggs of different sizes classified in XL (more than 73g), L (63 to $73 \mathrm{~g}$ ), M (53 to $63 \mathrm{~g}$ ), and S (until $53 \mathrm{~g}$ ) were used. In each test realized at the laboratory, one egg, randomly selected was illuminated with the projection of grids and the images were taken and processed to obtain the tridimensional shape of the object by moire technique (TM). The dimensions of its main axes were measured by means of a caliper rule, being used as a reference for the TM calibration. Empirical equations were adjusted for the correction of the dimensions, presenting coefficient of determination of 0.961, 0.955, and 0.988. TM showed to be adequate for recovering the egg topography providing errors smaller or equal to $5.47 \%$, with a clear demand of an independent calibration in the three dimensions, and without any standard object.
\end{abstract}

KEYWORDS: images analysis, egg, tridimensional shape.

\footnotetext{
${ }^{1}$ Eng $^{\mathrm{a}}$ Agrícola, Mestranda, Departamento de Engenharia, UFLA, Lavras - MG, Fone: 35-3829.1481, gleicedche@ hotmail.com.

${ }^{2}$ Doutor, Prof. Associado, Departamento de Engenharia, UFLA, Lavras - MG, yanagi@ deg.ufla.br.

${ }^{3}$ Prof. Fundação de Ensino Superior de Passos, FESP/UEMG, Doutorando, UFLA, Lavras - MG.

${ }^{4}$ Doutor, Prof. Associado, Departamento de Engenharia, UFLA, robbraga@ deg.ufla.br.

${ }^{5}$ Prof. Adjunto, Departamento de Engenharia, UFLA, Lavras - MG.

Recebido pelo Conselho Editorial em: 21-6-2010

Aprovado pelo Conselho Editorial em: 6-2-2011
} 


\section{INTRODUÇÃO}

O ovo representa um componente essencial na alimentação humana e na indústria. A casca do ovo é fonte de cálcio e substituto do carbonato de cálcio, que são produtos amplamente utilizados pelas indústrias alimentícias, farmacêuticas, odontológicas e de cosméticos (NAVES et al., 2007). A clara do ovo é fonte de lisozima e conalbumina, que são proteínas de alto valor nutricional e funcional (SOUSA et al., 2009). A albumina usada em suplementos alimentares controla a hipertensão e o ganho de massa muscular (SALVADOR \& SANTA, 2002). A gema constitui uma fonte de vitaminas (A, do complexo B, D e E), ácidos (palmítico, esteárico, oleico, linoleico, linolênico), cálcio, ferro, magnésio, sódio, selênio e zinco (NOVELLO et al., 2006).

A obtenção da geometria de ovos é de fundamental importância para estudos da agroindústria avícola e para estudos biológicos, tais como o desenvolvimento de pesquisas relacionadas à população e morfologia ecológica, para predizer a massa corporal de pintinhos, taxa de eclosão, características da qualidade da casca e parâmetros internos dos ovos (NARUSHIN, 2005). A obtenção da geometria de um ovo é relativamente complexa em condições experimentais, sendo que, no geral, medem-se as dimensões do menor e maior eixo e utilizam-se equações empíricas para determinação de outros parâmetros, tais como área superficial e volume do ovo (NARUSHIN, 2005; TRINDADE et al., 2007).

Para a recuperação da topografia de materiais biológicos por meio da análise de imagens, vários autores têm utilizado a técnica de moiré (TM), devido principalmente às vantagens de não requerer contato com o objeto, não ser um método destrutivo, apresentar rápida medição, alta sensibilidade, baixo custo e possibilidade de automatização (LINO \& DAL FABBRO, 2004; COSOLA et al., 2008; COSTA et al., 2008; LINO et al., 2008; RYU et al., 2008). ASSUNDI \& YUNG (1991) definem a TM como uma técnica versátil, baseada na interferência de grades superpostas, e que permite medir deformações de plano, contornos topográficos, perfis, declives, deformações, curvaturas e formas de objetos (COSTA et al., 2008).

Na medicina, a TM foi usada por GAO et al. (2006) na recuperação da topografia de dentes, por HAMRA \& VOLPON (1995) para avaliar quantitativamente o arco plantar mediar de pés adultos, por RODRIGUES et al. (2003) em desvios na coluna de crianças e adolescentes, e por RAHMAN et al. (2007) em estudos sobre perfuração da membrana timpânica. Na veterinária, BUYTAERT et al. (2009) usaram a TM para estudar a topografia dos tímpanos de coelhos. A projeção da TM ainda foi usada para criar modelos digitais de superfície de peras (LINO, 2002), determinar a área superficial de frangos de corte (SILVA, 2007), traçar a topografia de pedaços de madeira e de galinhas (COSTA et al., 2008), analisar esforços de compressão em bambu (BERALDO et al., 2007), reconstruir a superfície de uma maçã (BRAGA JÚNIOR et al., 2009) e visualizar linhas de força eletromagnéticas ao redor de duas cargas paralelas (YUK et al., 2008). Além disso, GOMES et al. (2009) propuseram uma metodologia de calibração aplicada à TM para investigações perfilométricas em protótipos mecânicos e ZHAO \& ZHANG (2006) concluíram que a TM tem aplicação em estudos mecânicos de objetos biológicos.

Desta forma, objetivou-se, com a presente pesquisa, desenvolver uma metodologia para a recuperação da forma tridimensional de ovos por meio de análise de imagens, utilizando a TM como metodologia de digitalização e a proposição de um modelo para a correção das distorções nas três dimensões do ovo.

\section{MATERIAL E MÉTODOS}

Ovos provenientes de galinhas da linhagem Hy-Line foram obtidos em uma granja comercial de postura, totalizando 15 unidades. Os ovos foram separados por meio de uma mesa densimétrica e classificados como XL (mais de $73 \mathrm{~g}$ ), L (63 a $73 \mathrm{~g}$ ), M (53 a $63 \mathrm{~g}$ ) e S (até $53 \mathrm{~g}$ ), sendo suas massas aferidas em laboratório por meio de uma balança digital com precisão de $\pm 0,05 \mathrm{~g}$. 
O arranjo experimental para aquisição de imagens foi composto por uma fonte de luz com potência de $150 \mathrm{~W}$ que projetava grades horizontais sobre o ovo, um anteparo vertical pintado de preto fosco e uma câmera fotográfica digital (Figura 1). A distância entre a câmera digital e o anteparo (Y) foi de $0,90 \mathrm{~m}$, e entre a câmera digital e a fonte de luz (X) foi de $0,53 \mathrm{~m}$.

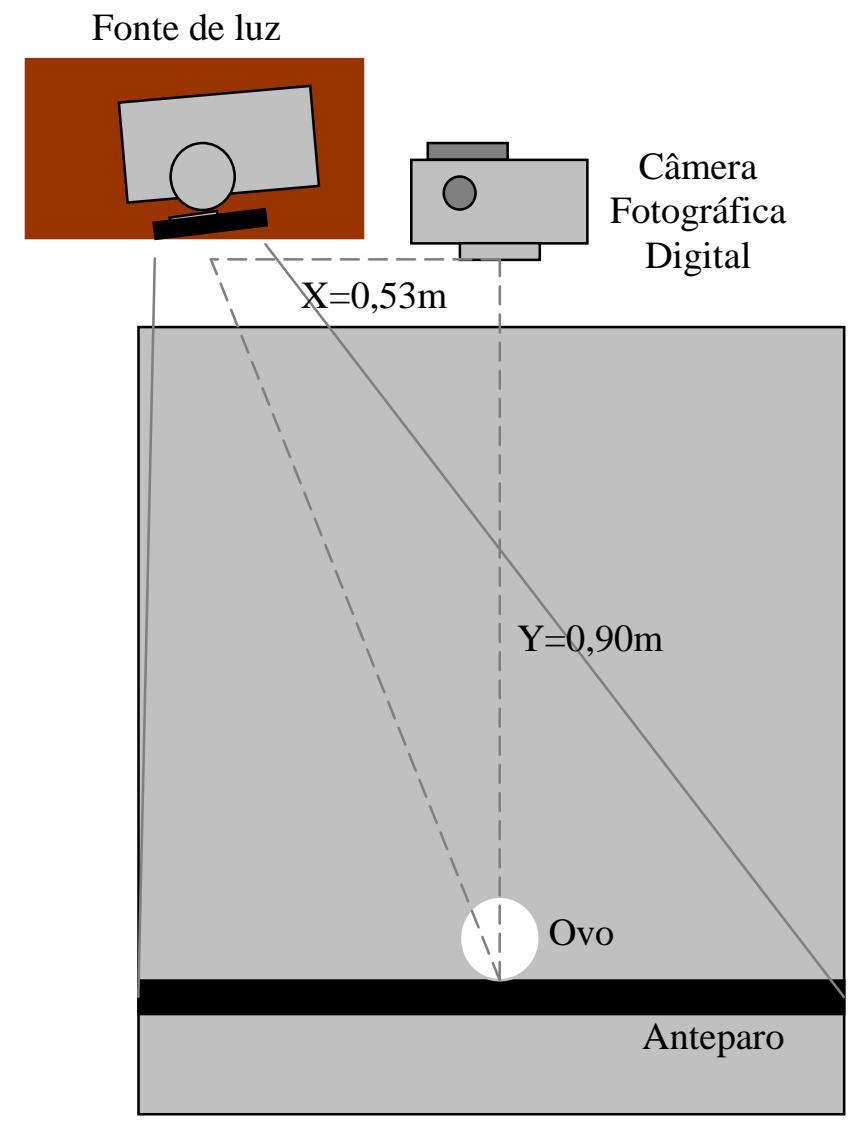

FIGURA 1. Arranjo experimental utilizado para a aquisição das imagens digitais. Experimental arrangement used for acquisition of the digital images.

A projeção da grade pela fonte luminosa no anteparo abrangeu uma área de $0,08 \mathrm{~m}^{2}(0,26 \mathrm{~m}$ de altura e $0,30 \mathrm{~m}$ de comprimento). A distância entre o anteparo e o ovo foi zero (0), e o ovo foi colocado perpendicularmente à projeção da tela para um bom contraste de imagens. Todas as imagens dos ovos, independentemente das massas, foram obtidas ajustando a resolução da câmera fotográfica digital no modo VGA e o zoom de 2,0 vezes, correspondendo a uma área de captura de imagem de $0,18 \mathrm{~m}^{2}$ (0,40 $\mathrm{m}$ de altura e $0,45 \mathrm{~m}$ de comprimento). Estes valores de resolução e de zoom foram definidos em função do tamanho do objeto (ovo), da qualidade das imagens e da capacidade de processamento dos programas utilizados.

As grades foram geradas usando o programa Autocad ${ }^{\circledR} 2006$ (AUTODESK, 2005), com linhas de grade de espessura de $0,25 \mathrm{~mm}$ e espaçadas em 0,65 $\mathrm{mm}$, sendo impressas em folha transparente por uma impressora a laser, com resolução de 600 dpi. De acordo com COSTA et al. (2008), o ajuste das grades depende do arranjo experimental, das distâncias e dos ângulos. Estas grades, projetadas no ovo, caracterizaram o retículo principal, sendo, em seguida, projetadas no anteparo, sem o ovo, gerando o retículo de referência.

As imagens capturadas foram processadas usando o programa Microsoft ${ }^{\circledR}$ Paint, pelo processo de phase shift, no qual o número de pixels existentes no intervalo entre uma franja e outra era contado, e esse valor, dividido por quatro, representava a quantidade de pixels necessária a cada deslocamento para gerar um novo plano. Dessa forma, foram gerados quatro planos defasados de $1 / 4$, sendo o primeiro deslocamento o próprio plano (SILVA, 2007). 
Essas imagens pré-processadas eram introduzidas em uma função de dados desenvolvida na plataforma Scilab ${ }^{\mathrm{TM}}$ para a geração da imagem tridimensional do ovo. Neste processo, foi utilizada a caixa de ferramentas Scilab Image Processing, obtida no endereço http://sourceforge.net/projects/siptoolbox.

O programa Surfer ${ }^{\circledR}$ (Golden Software, Inc.) foi utilizado para a determinação das dimensões dos eixos do ovo. Inicialmente, dados gerados pelo Scilab ${ }^{\mathrm{TM}}$ de coordenadas x (maior dimensão), y (menor dimensão 1) e z (menor dimensão 2) do ovo foram importados ao programa Surfer ${ }^{\circledR}$. Então, foi usado o comando Grid - Data para selecionar o arquivo gerado na etapa anterior e proceder ao teste estatístico das colunas $\mathrm{x}, \mathrm{y}$ e $\mathrm{z}$.

A partir destes dados, foi gerado um gráfico de isocotas com uma superfície ao redor do ovo. Esse excesso de superfície foi removido do mapa por meio do comando Digitize, restando, assim, somente a imagem tridimensional do ovo. Nesta imagem, foram medidas as dimensões do ovo (maior dimensão, menor dimensão 1 e menor dimensão 2) pela ferramenta Polyline do programa Surfer ${ }^{\circledR}$.

O método convencional para a determinação das dimensões de cada ovo foi usado como uma referência para calibrar o método baseado na TM. No método convencional, cada ovo teve sua maior dimensão e menor dimensão aferidas por meio de um paquímetro manual com precisão de $0,05 \mathrm{~mm}$.

Inicialmente, na calibração da TM, que abrangeu ovos classificados em todas as categorias de tamanho, foram processadas as imagens sem aplicação dos fatores de correção. Após a análise de resultados, verificou-se a necessidade de se ajustar equações empíricas para a correção das dimensões, sendo que todas as imagens foram processadas novamente, aplicando-se estes fatores. As dimensões estimadas pela TM e corrigidas pelas equações empíricas ajustadas foram comparadas aos valores determinados pelo método convencional, por meio do teste t.

\section{RESULTADOS E DISCUSSÃO}

Após a análise preliminar das imagens processadas, verificou-se a necessidade de correção das dimensões dos eixos principais do ovo. Desta forma, foram ajustadas três equações para determinar os fatores de correção para a maior dimensão ( $\left.\mathrm{F}_{\mathrm{C}, \text { Dmaior }}\right)$ [eq.(1)], menor dimensão 1 $\left(\mathrm{F}_{\mathrm{C}, \text { Dmenor1 }}\right)$ [eq.(2)] e menor dimensão 2 ( $\left.\mathrm{F}_{\mathrm{C}, \text { Dmenor2 }}\right)$ [eq.(3)], em função das dimensões do ovo obtidas por meio das imagens processadas pelo programa Surfer ${ }^{\circledR}$, apresentando coeficientes de determinação iguais a 0,9610; 0,9675 e 0,9986, respectivamente. O ajuste das equações de calibração está representado na Figura 2.

Na Figura 3, ilustra-se a recuperação da forma tridimensional do ovo, antes (a) e após (b) aplicar o fator de correção. Os resultados obtidos nesta pesquisa confirmam a capacidade da TM de recuperar adequadamente a forma tridimensional de materiais biológicos, tais como a de uma pera (LINO, 2002), de maçãs (BRAGA JÚNIOR et al., 2009), de frangos de corte (SILVA, 2007) e de galinhas (COSTA et al., 2008).

$$
\begin{aligned}
& \mathrm{F}_{\mathrm{C}, \text { Dmaior }}=-2 \times 10^{-6} \mathrm{D}_{\text {maior }}{ }^{3}+0,0007 \mathrm{D}_{\text {maior }}{ }^{2}-0,0776 \mathrm{D}_{\text {maior }}+3,4839 \\
& \mathrm{~F}_{\mathrm{C}, \text { Dmenor } 1}=22,121 \mathrm{D}_{\text {menor } 1}{ }^{-0,8215} \\
& \mathrm{~F}_{\mathrm{C}, \text { Dmenor } 2}=43,209 \mathrm{D}_{\text {menor } 2}{ }^{-0,8966}
\end{aligned}
$$


(a)



(b)

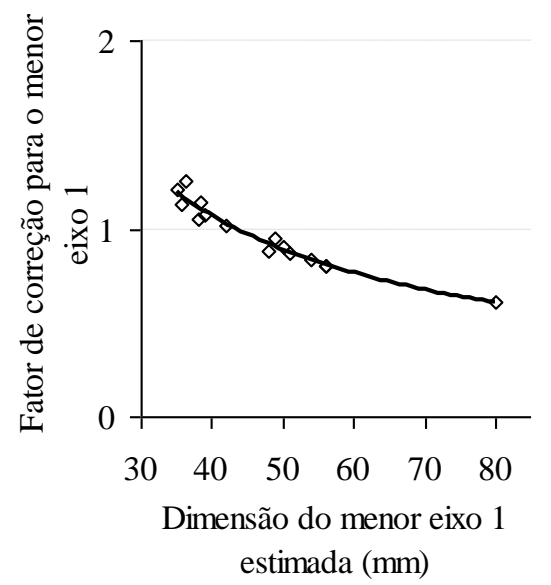

(c)

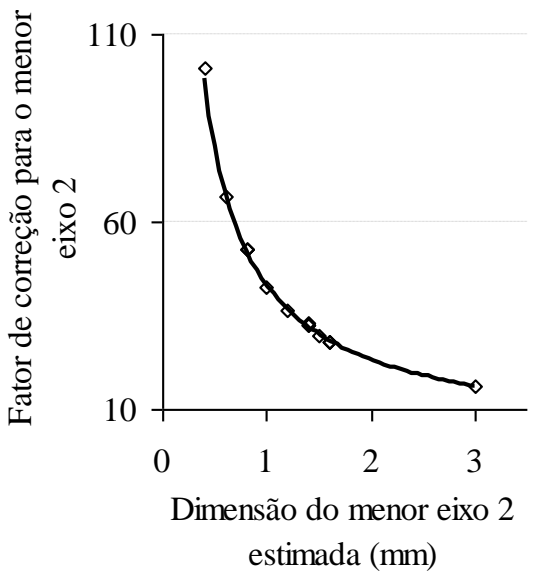

FIGURA 2. Ajuste dos fatores de correção para: (a) maior dimensão, (b) menor dimensão 1 e (c) menor dimensão 2. Adjust of the correction factors for the (a) larger dimension, (b) smaller dimension 1 and (c) smaller dimension 2.

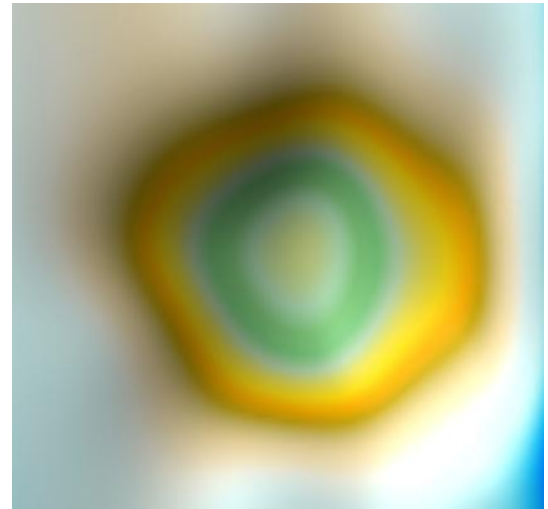

(a)



(b)

FIGURA 3. Representação da imagem tridimensional do ovo: (a) antes da correção e (b) depois da correção, gerados pelo programa Surfer ${ }^{\circledR}$. Representation of the three-dimensional image of the egg (a) before the correction and (b) after the correction, generated by the Surfer ${ }^{\circledR}$ program.

Após a calibração, verificou-se que não existem diferenças estatísticas ( $\mathrm{P}>0,05$, teste $\mathrm{t}$ ) entre os valores das dimensões estimadas pela TM e os medidos pelo paquímetro. As relações funcionais entre as dimensões são ilustradas nas Figuras $4 a, 4 b$ e $4 c$, sendo que os valores de desvio-padrão das médias foram de 1,$878 ; 1,457$ e $1,743 \mathrm{~mm}$, e os erros percentuais, de $5,47 \% ; 2,60 \%$ e 3,42\%, 
respectivamente. SILVA et al. (2010), ao estudarem a recuperação da forma tridimensional de frangos de corte por meio da TM, verificaram que os erros de calibração e validação foram de $3,4 \%$ e 5,18\%, respectivamente, quando comparados a um método convencional destrutivo. COSTA et al. (2008), estudando a deformação de madeira pela TM, verificaram a habilidade da técnica para identificar deformações de $0,4 \mathrm{~mm}$.

De acordo com DIRCKX et al. (2010), a altura de pequenos objetos pode ser obtida com uma precisão de $15 \mu \mathrm{m}$. GOMES et al. (2009) determinaram um erro de $0,98 \%$ ao recuperar o perfil de um mouse pela TM, evidenciando uma forma de calibração em apenas uma dimensão, o que foi claramente descartada neste trabalho, uma vez que não seria suficiente para as três dimensões do ovo, que apresentaram modelos de correções não linear. A proposta de calibração apresentada neste trabalho mostra uma vantagem daquela proposta por GOMES et al. (2009), que usaram um objeto calibrador, o que não se fez necessário neste caso.

(a)

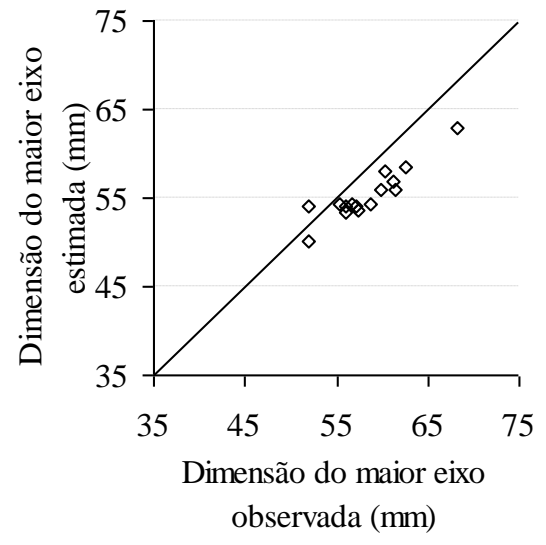

(b)

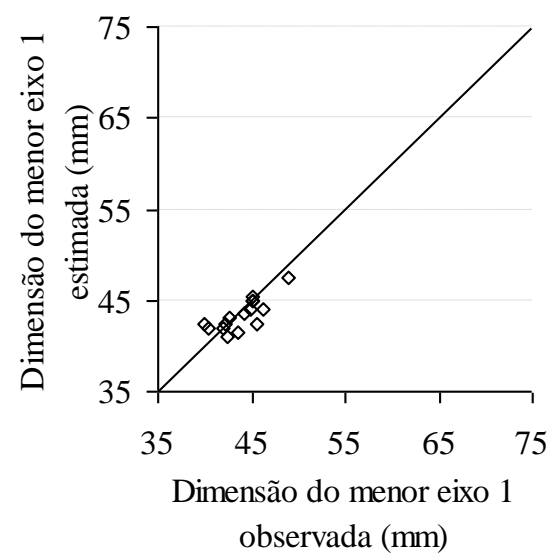

(c)



FIGURA 4. Relação funcional entre: (a) maior dimensão, (b) menor dimensão 1 e (c) menor dimensão 2 medidas e estimadas pela TM. Functional relationship among the (a) largest dimension, (b) smaller dimension 1 and (c) smaller dimension 2 measures and estimated by TM.

\section{CONCLUSÕES}

A técnica de moiré, associada à análise de imagens, mostrou-se adequada para a recuperação da topografia de ovos, proporcionando erros menores ou iguais a 5,47\%, evidenciando a necessidade da criação de um modelo de correção das distorções nas três dimensões do ovo e sem um objeto calibrador. 


\section{AGRADECIMENTOS}

À FAPEMIG, CAPES e CNPq, pelo suporte financeiro a esta pesquisa.

\section{REFERÊNCIAS}

ASSUNDI, A.; YUNG, K. H. Logical moiré and its application. Experimental Mechanics, London, v.31, n.3, p.236-242, 1991.

AUTODESK. Autodesk inventor series: getting started. San Rafael: Autodesk, v.10, 2005. 286 p.

BERALDO, A.L.; ALBIERO, D.; MACIEL, A.J.S.; DAL FABBRO, I.M.; RODRIGUES, S. Técnica de moiré aplicada al análisis de esfuerzos de compresión en el bambú guadua. Ciencia y tecnología, Maputo, v.9, n.3, p.309-322, dic. 2007.

BRAGA JÚNIOR, R.A.; OLIVEIRA, B.S.; COSTA, R.M.; LINO, A.C.L.; DAL FABBRO, I.M. Suppression of border effects in moiré techniques using three-dimensional methods. Biosystems Engineering, Harpenden, v.102, n.1, p.1-8, jul. 2009.

BUYTAERT, J.A.N.; AERNOUTS, J.E.F.; DIRCKX, J.J.J. Indentation measurements on the eardrum with automated projection moiré profilometry. Optics and Lasers in Engineering, Oxford, v.47, n.3-4, p.301-309, 2009.

COSOLA, E.; GENOVESE, K.; LAMBERTI, L.; PAPPALETTERE, C. A general framework for identification of hyper-elastic membranes with moiré techniques and multi-point simulated annealing. International Journal of Solids and Structures, Nova York, v.45, n.24, p.6.074-6.099, dez. 2008.

COSTA, R.M.; BRAGA JUNIOR, R.A.; OLIVEIRA, B.S.; SILVA, E.; YANAGI JUNIOR, T.; LIMA, J.T. Sensitivity of the moiré technique for measuring biological surfaces. Biosystems Engineering, Harpenden, v.100, n.3, p.321-328, jul. 2008.

DIRCKX, J.J.J.; BUYTAERT, J.A.N.; VAN DER JEUGHT, S.A.M. Implementation of phaseshifting moiré profilometry on a low-cost commercial data projector. Optics and Lasers in Engineering, Oxford, v.48, n.2, p.244-250, feb. 2010.

GAO, J.; XU, W.; GENG, J. 3D shape reconstruction of teeth by shadow speckle correlation method. Optics and Lasers in Engineering, Oxford, v.44, n.5, p.455-465, may 2006.

GOMES, T.S.; BRAGA JÚNIOR, R.A.; LINO, A.C.L.; RABELO, G.F.; COSTA, R.M. Calibração da técnica de moiré aplicada à perfilometria de protótipos mecânicos. Ciência e Agrotecnologia, Lavras, v.33, n.2, p.574-579, mar./abr. 2009.

HAMRA, A.; VOLPON, J.B. Fotopodometria "moiré" quantitativa na avaliação do arco plantar longitudinal medial. Revista Brasileira de Ortopedia, São Paulo, v.30, n.8, p.609-614, ago. 1995.

LINO, A.C.L.; DAL FABBRO, I.M. Determinação da topografia de uma fruta pela técnica de moiré de sombra com multiplicação de franjas. Ciência e Agrotecnologia, Lavras, v.28, n.1, p.119125, jan./fev. 2004.

LINO, A.C.L.; SANCHES, J.; DAL FABBRO, I.M. Image processing techniques for lemons and tomatoes classification. Bragantia, Campinas, v.67, n.3, p.785-789, 2008.

LINO, A.C.L. Técnica óptica de moiré visando à aplicação no estudo de superfícies irregulares. 2002. 85 f. Dissertação (Mestrado em Engenharia Agrícola) - Universidade Estadual de Campinas, Campinas, 2002.

NARUSHIN, V. G. Egg geometry calculation using the measurements of length and breadth.

Poultry Science, Champaign, v.84, n.3, p.482-484, mar. 2005. 
NAVES, M.M.V.; PRADO, C.M.M.; FERNANDES, D.C.; SERAFINI, A.B. Avaliação microbiológica do pó da casca de ovo e otimização da técnica de elaboração do produto. Pesquisa Agropecuária Tropical, Goiânia, v.37, n.2, p.113-118, jun. 2007.

NOVELLO, D.; FRANCESCHINI, P.; QUINTILIANO, D.A.; OST, P.R. Ovo: conceitos, análises e controvérsias na saúde humana. ALAN, Caracas, v.56, n.4, p.315-320, dez. 2006.

RAHMAN, A.; UNGE, M. Von; OLIVIUS, P.; DIRCKX, J.; HULTCRANTZ, M. Healing time, long-term result and effects of stem cell treatment in acute tympanic membrane perforation. International Journal of Pediatric Otorhinolaryngology, Nova York, v.71, n.1, p.1.129-1.137, jul. 2007.

RODRIGUES, L.F.; FERNANDES, M.; BARROS, J.W.; SHIMANO, A.C.; MOREIRA, F.B.R.; GONÇALVES, F.F.; AMORIM, G.S.; OTONI, N.T.; RODRIGUES, S.A; PINTO, T.A.; SANTOS, V.C. Utilização da técnica de moiré para detectar alterações posturais. Revista de Fisioterapia, São Paulo, v.10, n.1, p.16-23, jan./jun. 2003.

RYU, W-J.; KANG, Y-J.; BAIK, S-H.; KANG, S-J. A study on the 3-D measurement by using digital projection moiré method. Optik - International Journal for Light and Electron Optics, Amsterdam, v.119, n.10, p.453-458, ago. 2008.

SALVADOR, M.; SANTA, P.D. Teores de macronutrientes e de colesterol em diferentes tipos de ovos. Boletim CEPPA, Curitiba, v.20, n.1, p.133-140, jan./jun. 2002.

SILVA, E. Desenvolvimento e validação de um modelo matemático para o cálculo da área superficial de frangos de corte. 2007. 59 f. Dissertação (Mestrado em Engenharia Agrícola) Universidade Federal de Lavras, Lavras, 2007.

SILVA, E.; YANAGI JUNIOR, T.; BRAGA JÚNIOR, R.A.; LOPES, M.A.; DAMASCENO, F.A.; SILVA, G.C. de A. e S. Digital surface area assessment of broiler chickens. Biosystems Engineering, London, 2010. In Review.

SOUSA, R.C.S.; COIMBRA, J.S.R.; SILVA, L.H.M.; SILVA, M.C.H.; ROJAS, E.E.G.; VICENTE, A.A.M.O.S. Thermodynamic studies of partitioning behavior of lysozyme and conalbumin in aqueous two-phase systems. Journal of Chromatography B, Amsterdam, v.877, n.24, p.2.579-2.584, ago. 2009.

TRINDADE, J.L.; NASCIMENTO, J.W.B.; FURTADO, D.A. Qualidade de ovo de galinhas poedeiras criadas em galpões no semiárido paraibano. Revista Brasileira de Engenharia Agrícola e Ambiental, Campina Grande, v.11, n.6, p.652-657, nov./dez. 2007.

YUK, K.C.; LEE, T.H.; CHANG, S. A moiré method of visualizing electromagnetic force lines. Optik - International Journal for Light and Electron Optics, Amsterdam, v.119, n.10, p.473-476, ago. 2008.

ZHAO, Y.; ZHANG, X. Determination of the deformations in polymeric nanostructures using geometric moiré techniques for biological applications. Sensors and Actuators B: Chemical, Lausanne, v.117, n.2, p.376-383, out. 2006. 